Session 1559

\title{
PLC Stepper Motor Controller
}

\section{Leonard Sokoloff \\ DeVRY Institute}

In this application PLC is used to control the operation of a stepper motor. Ladder Logic Diagram, the PLC program, uses step ladder instructions for implementation of the control algorithm. The motion control algorithm includes the control of stepper motor speed and direction of rotation. The stepper motor is a four phase permanent magnet type.

The interface board is designed to generate appropriate voltage and current levels for each phase of the stepper motor. Darlington transistors with inductive kick protection are used for this purpose.

The Programmable Logic Controller (PLC) is a relatively new technology that uses a computer to process the information. The control task is incorporated into a graphical program called the Ladder Logic Diagram. Any control task modifications are done by changing the program. This is why the use of the PLC is preferred to the traditional hard wired circuits in industrial controls.

The traditional hard-wired control circuits are designed to perform a dedicated task. A temperature control board, for example, accepts its inputs from temperature sensors, processes these signals, and generates appropriate control signals to turn ON or OFF output devices such as heaters, fans, or air conditioners. All hard-wired circuits are generally very costly to design and build. If a circuit has to be modified, it must be torn apart and rewired, thus making modifications very costly.

This application is part of an effort at DeVry to include into the curriculum important technologies such as the virtual instrumentation and the programmable logic controllers. The PLC Stepper Motor Controller is one of several projects that are being added to the upper trimester laboratory. 


\section{Stepper Motor Operation}

The shaft of a stepper motor rotates one step at a time. This type of motion is called a discrete motion. The step angle is determined from

$$
\Theta=360 / \mathrm{SR}
$$

where SR is the stepping rate in steps per revolution. If SR, for example, is equal to 200 then from the above equation, the step angle is $1.8^{\circ}$. Stepper motors are known for a very accurate motion, and for that reason are used in applications such as a floppy disk drive. The operation of a stepper motor can be understood from the illustration in Fig. 1. The stepper motor here has four phases labeled as $\phi 1$ through $\phi 4$. A phase is a winding with many turns of wire on one or more poles of the stator.

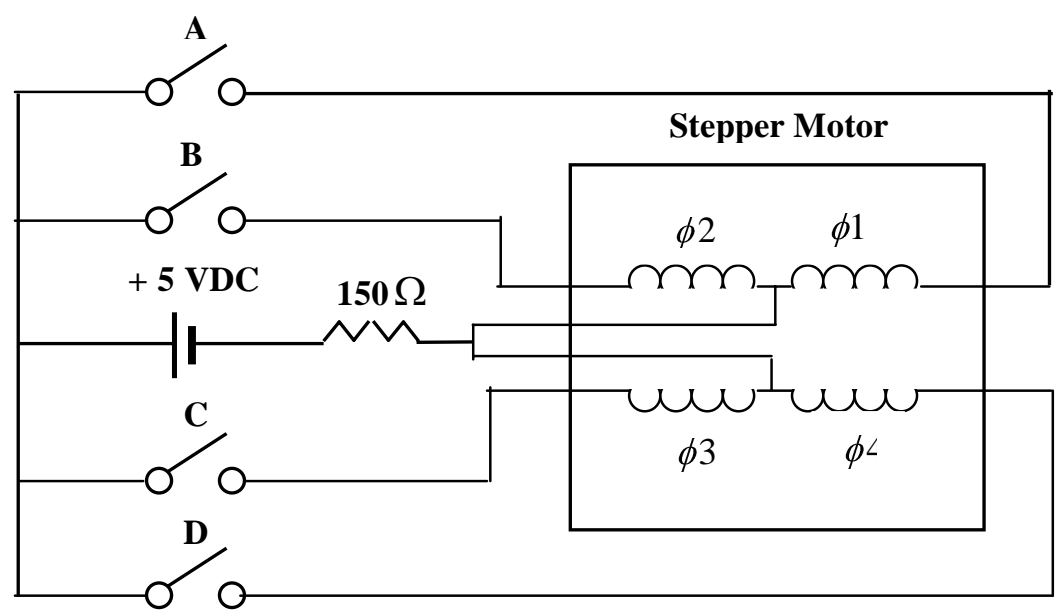

\begin{tabular}{|c||c|c|c|c|}
\hline$\Theta$ & $\mathbf{A}$ & $\mathbf{B}$ & $\mathbf{C}$ & $\mathbf{D}$ \\
$\phi 1$ & $\phi 2$ & $\phi 3$ & $\phi 4$ \\
\hline \hline $\mathbf{0}$ & $\mathbf{X}$ & & & \\
\hline $\mathbf{1 . 8}$ & & $\mathbf{X}$ & & \\
\hline $\mathbf{3 . 6}$ & & & $\mathbf{X}$ & \\
\hline $\mathbf{5 . 4}$ & & & & $\mathbf{X}$ \\
\hline $\mathbf{7 . 2}$ & $\mathbf{X}$ & & & \\
\hline $\mathbf{9 . 0}$ & & $\mathbf{X}$ & & \\
\hline
\end{tabular}

Fig. 1 The Driving Circuit for a Stepper Motor. 
To excite the phase of a stepper motor, an appropriate current specified by the manufacturer must be applied. If the toggle switch A in Fig. 1, for example, is closed, current will flow in the winding of $\phi 1$. When switch A is opened and switch B is closed, the current is now switched to the $\phi 2$ winding, causing the motor to make a $1.8^{\circ}$ step. We will now excite $\phi 3$ by opening switch $\mathrm{B}$ and closing switch $\mathrm{C}$, thus forcing the motor to execute another $1.8^{\circ}$ step. The motor will step once more after we continue with this sequence of opening switch $\mathrm{C}$ and closing switch $\mathrm{D}$. The phase excitation sequence, as illustrated in the table of Fig. 1 is assumed to be 1-2-3-4 (the " $X$ " indicates the phase being excited). This is the required sequence for clockwise rotation. For counterclockwise rotation, the sequence must be reversed, or 4-3-2-1 sequence must be applied. The sequence for a given direction of rotation is specified by the manufacturer. The motor will continue stepping as long as the sequence is repeated.

Manual operation of the toggle switches does work, however the motor will be stepping very slowly. Since an average stepper motor is capable of hundreds of steps per second in a practical application, a better method of driving the stepper motor must be devised.

Fig. 2 shows a typical stepper motor driving circuit. The toggle switches used in Fig. 1 are replaced here with Darlington amplifiers. Darlington circuits are used here not only to switch the currents among the four phases, but also to provide the required current gain. Depending on the size of the stepper motor, few tens, or a few hundreds, or perhaps, in a case of a large stepper motor, few amperes of current may be required to drive it. The pulses, shown in the illustration, are generated by an electronic circuit elsewhere. Actually, in this experiment, they originate in the PLC equipment.

The 1 N4001 diode in series with the $100 \Omega$ resistor is in parallel with the phase winding. It the diode-resistor combination that suppresses the inductive kick. Inductive kick occurs when the current through the phase winding is suddenly interrupted when the Darlington turns OFF on the trailing edge of the input pulse. If it wasn't for the diode that becomes forward biased at this time, providing a discharge path for the current, the Darlington transistor would be destroyed from over-voltage.

Fig. 3 shows the timing diagram for phase excitation. During time T1 the current is applied only to phase 1 . Then phase 2 is excited during T2, followed by the excitation of phases 3 and 4 . The cycle is repeated if the stepper motor is to continue the rotation. 


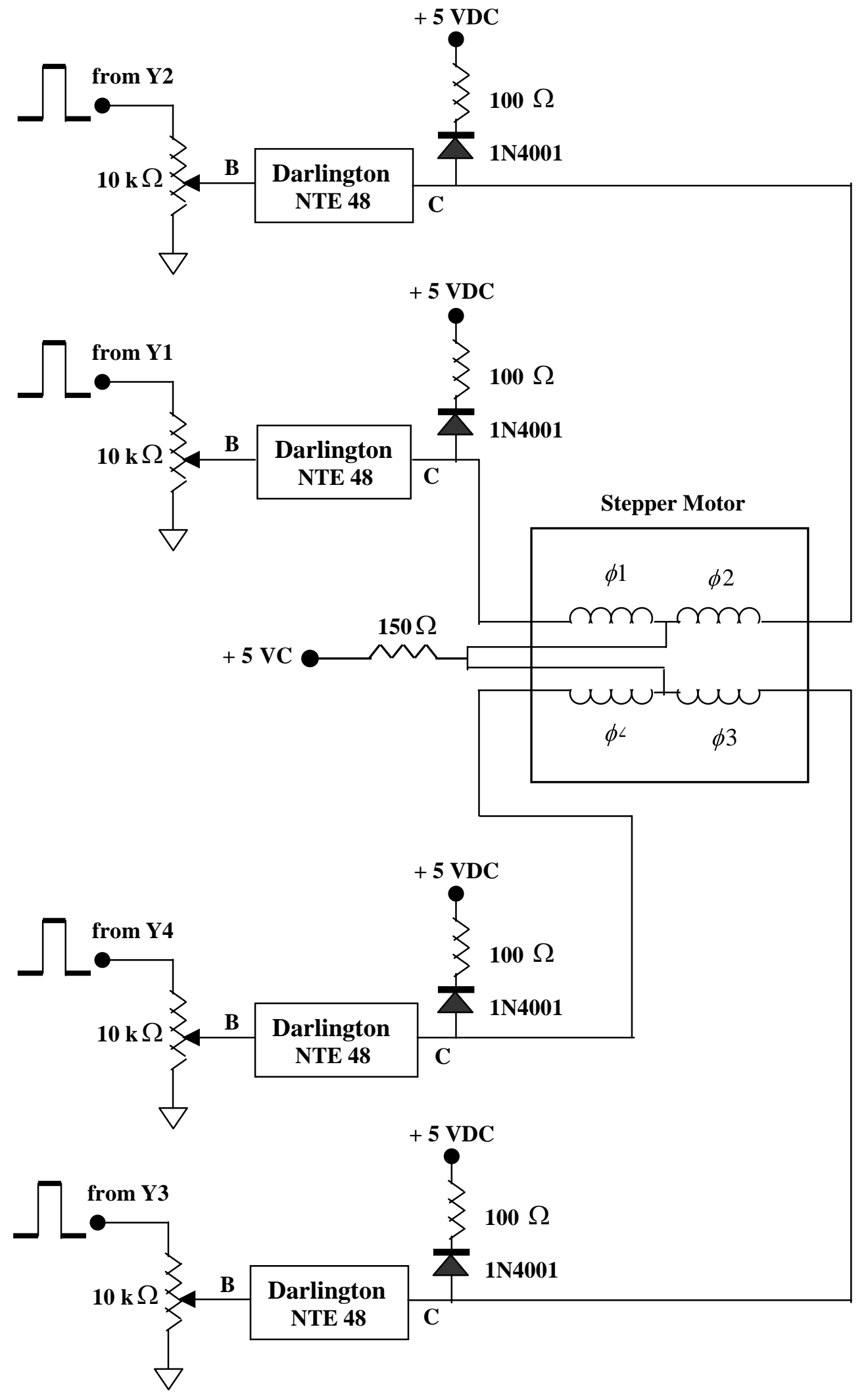

Fig. 2 Darlington Drivers for the Stepper Motor. 


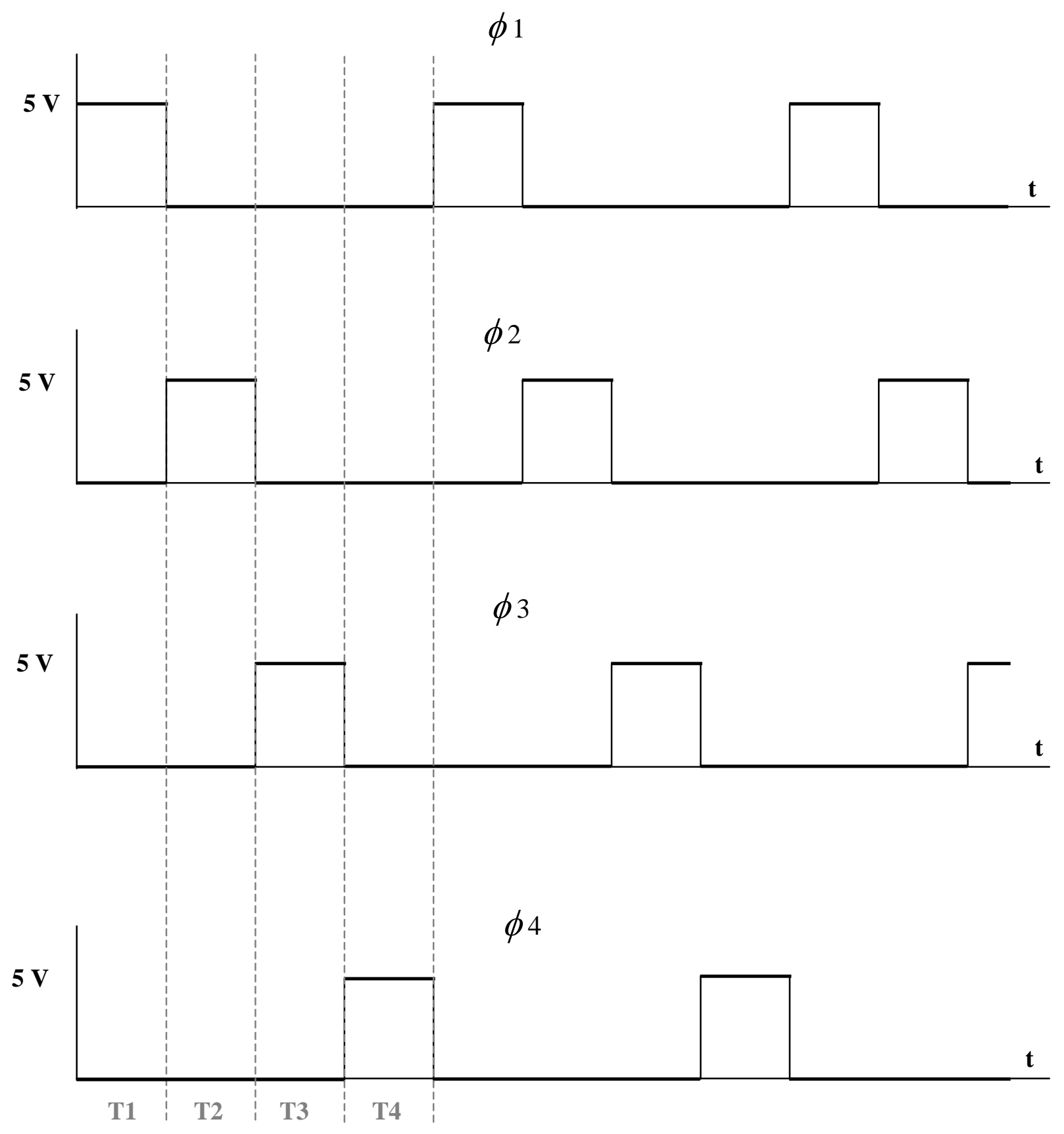

Fig. 3 Phase Excitation Timing Waveforms. 


\section{System Hardware}

\section{Stepper Motor Controller}

The hardware used in this experiment is illustrated in Fig. 4. The PLC system is plugged into the computer's communication port. The interface or the driver board includes the signal conditioning circuits as shown in Fig. 2. It receives control pulses from PLC's Output Module. These pulses are conditioned on the driver board and then applied to the individual phases of the stepper motor. Switches X1 and X2 on the Input Module are used for controlling the motion of the stepper motor. X1 controls the direction of rotation and X2 controls the speed. The operator can select one of two operating speeds. Each speed is associated with the state of the X2 switch and is under software control.

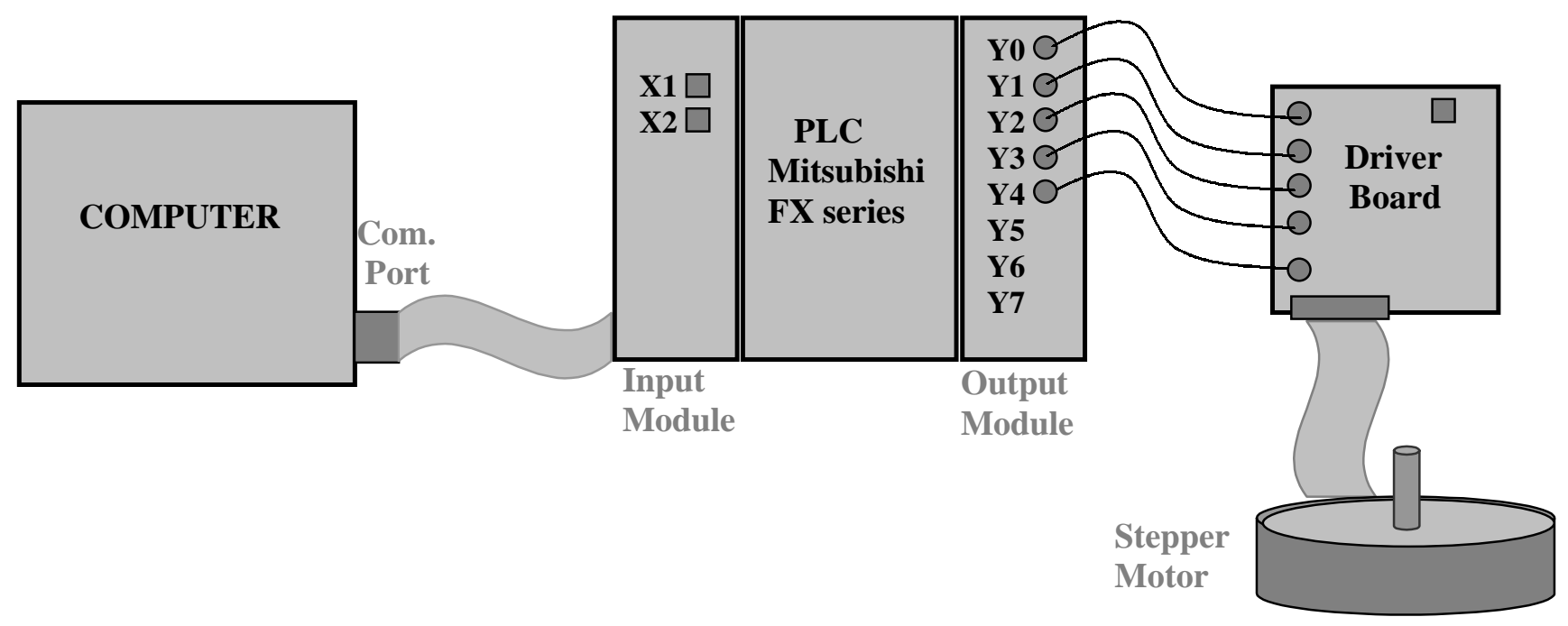

Fig. 4 Hardware Configuration for the Stepper Motor Controller. 


\section{System Software}

In PLC environment, a program is implemented in what is known as the Ladder Logic Diagram. It is called that because its shape resembles a ladder. The inputs which are received from the Input Module are called contacts and the outputs called coils are on the Output Module of the PLC system. The outputs are used to drive various output devices. Each rung of the Ladder Logic Diagram contains contacts on the left side and coils on the right side. The PLC syntax is quite extensive including arithmetic, logical, branching and other operations.

In this experiment, however, Step Ladder instructions are used to provide the sequential control to drive the stepper motor. The step ladder is a special relay function that provides a sequential activation of various output elements. Step Ladder uses state relays as illustrated in the following partial diagram.

The two vertical lines in the Ladder Logic Diagram are called rails representing the two sides of the power supply AC or DC. The PLC used in this experiment is DC based.

On each Scan Cycle the system reads the state of the input devices that are connected to the Input Module and updates the Input Image Table in memory. Then it executes user's program (Ladder Logic Diagram also located in memory) and updates the Output Image Table. As the last step, the system activates or deactivates various output devices that are connected to the Output Module of the PLC. The Scan Cycle is repeated several thousand times each second.

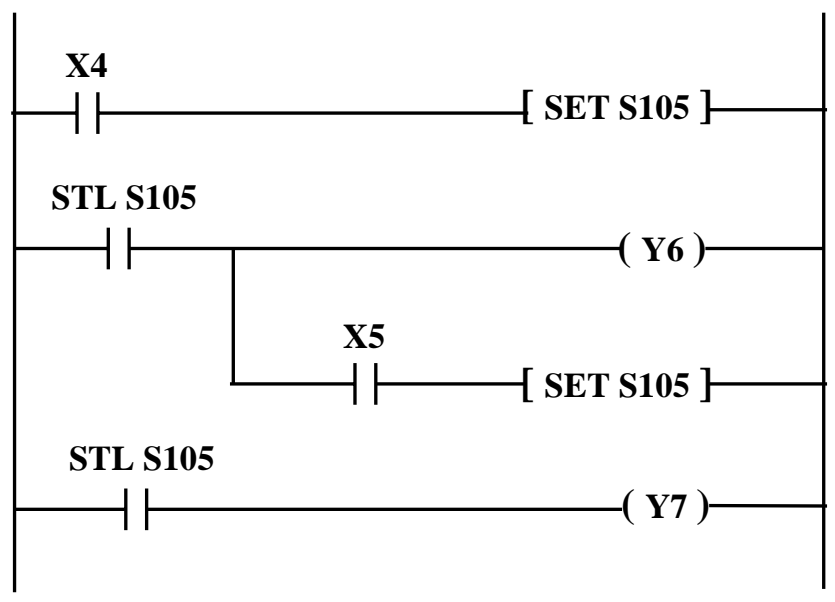

When X4 is turned ON, its contact is energized and SET S105 state relay instruction is executed. SET is the command that activates the state relay. This action energizes the STL S105 contact applying power to Y6 output, thus activating its coil. Y6 is a terminal that is located on the Output Module of the PLC and if an external device was connected to Y6, then power would be applied to that device. 
Should X5 be energized at this time, that will activate S105 state relay. This action will reset S104, de-energizing contact STL S104 and at the same time energizing STL S105 contact thus turning OFF Y6 and turning ON Y7. This property of the state relay where an energized state relay turns OFF the preceding state relay is very useful in sequential applications. The use of the Step Ladder instructions results in an efficient program with fewer programming steps.

A quick inspection of Ladder Logic Diagram shown in Fig. 5 suggests that it has four sectors of programming activity. Each sector is enabled by an STL contact which in turn is energized by the corresponding state relay. The four sectors resemble each other in format and the type of task that they perform. Perhaps a more descriptive view of the tasks performed by each sector is revealed by the flow chart shown in Fig. 6.

Turning to the flow chart of Fig. 6, the reader will note that the operation begins by turning ON the Start switch. When it is ON, state relay S100 is activated and the corresponding contact STL S100 is energized. This enables the first sector where two input toggle switches are tested to determine their state. X1 controls the direction of rotation, clock-wise if $\mathrm{ON}$ and counter-clockwise if it is turned OFF. X2, on the other hand determines the motor speed. When X2 is ON, a 1 second timer T200 is activated and when it is OFF a $20 \mathrm{msec}$ timer T201 is activated.

Assuming that $\mathrm{X} 1$ is $\mathrm{ON}$, power is applied to the first phase winding $\phi 1$ of the stepper motor, since YO coil is wired to drive $\phi 1$, for the duration of time as determined by X2 and one of the two timers. The 1 second timer sets the slow speed while the $20 \mathrm{msec}$ timer, the fast speed. The ON state of X1 initiates the clock-wise rotation. Regardless of which timer is activated, at the end of its timing cycle the corresponding contact (T200 or T201) is energized and the state relay S101 is activated. This action results in contact STL S101 being energized thus enabling the second sector and disabling the preceding sector.

Assuming that the state of the switches $\mathrm{X} 1$ and $\mathrm{X} 2$ has not changed, two tasks will be executed in this sector. First, the second phase $\phi 2$ will be powered (power was removed from the first phase when S101 was activated disabling the first sector). Second, S102 state relay will be activated, thus disabling the present (second sector and enabling the third).

This sequential action continues until in the fourth sector S100 state relay is set and the Return command is encountered. The sequence is now repeated from the first sector and will repeat indefinitely until the Run switch is set to OFF. The flow chart highlights the individual steps of the clock-wise and counter clock-wise motions. Clock-wise on the right-hand side and counter-clockwise on the left-hand side. 


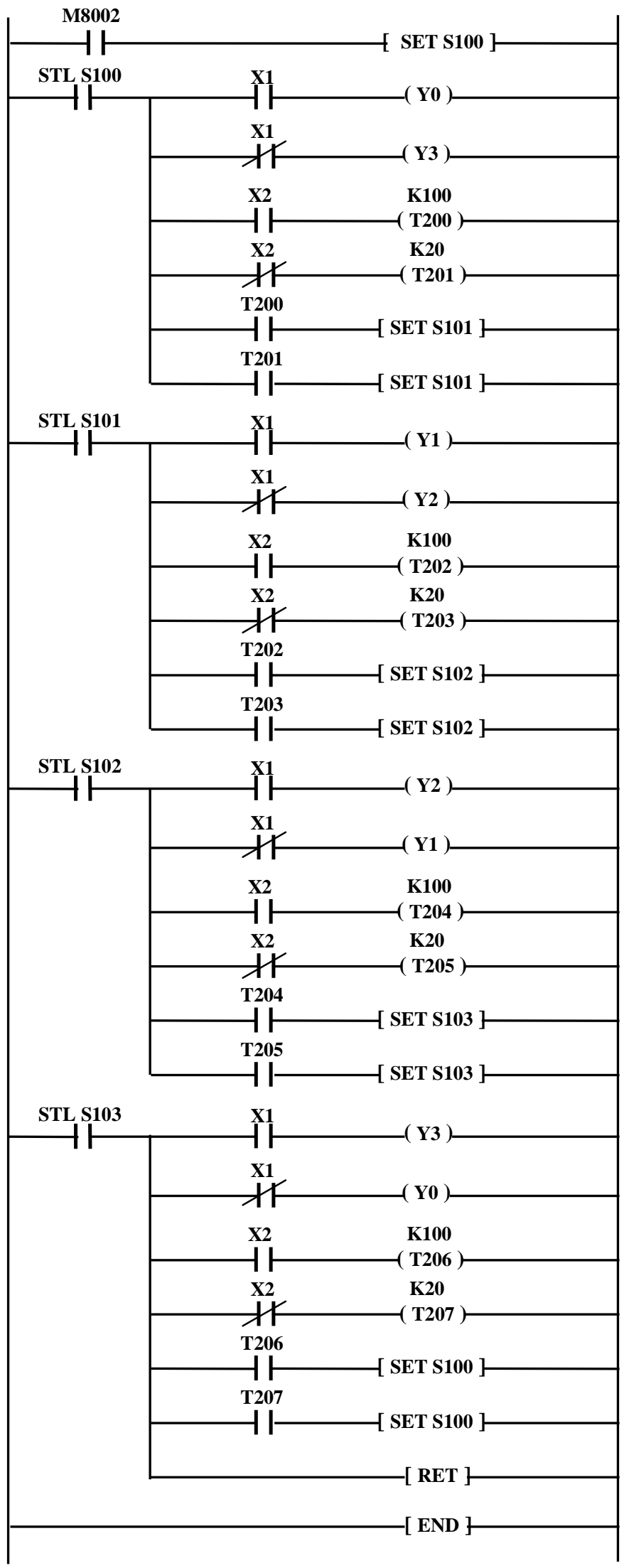

Fig. 5 The Stepper Motor Controller Program. 


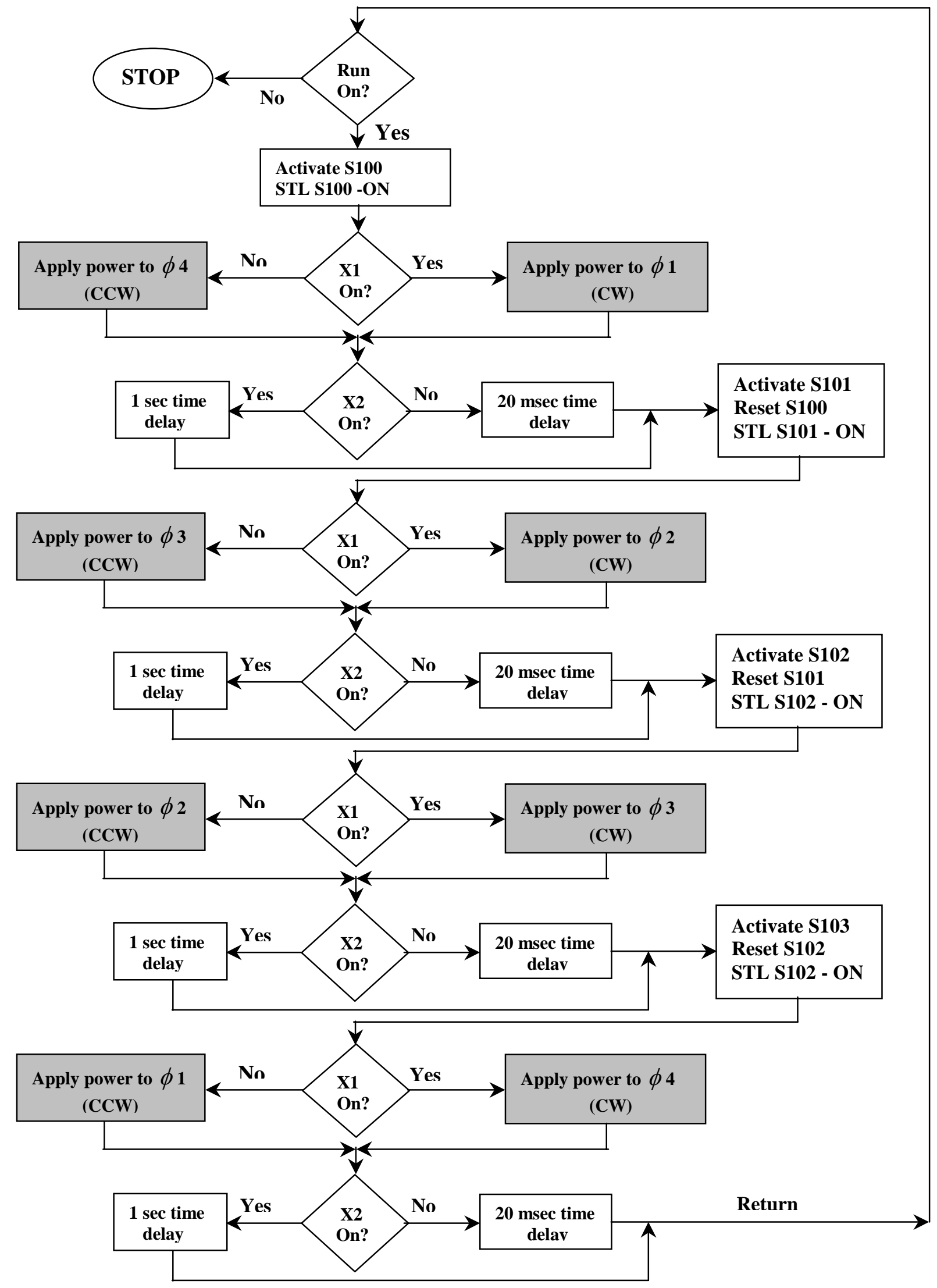

Fig. 6 The Stepper Motor Controller Flow Chart 


\section{Conclusion}

The stepper motor controller was tested and the results show that its operation is consistent with the theory described in this article.

Switch X2 controls the speed of the motor. Because one only switch was used, only two speeds are possible The software can be modified to include more than one switch and thus provide the capability of controlling more than two speeds.

In order to view the stepping motion of the motor, the speeds used in the experiment were somewhat slow. The motor speed, however, can be adjusted by choosing another $\mathrm{K}$ value of the timer. The $\mathrm{K}$ value specifies the number of timing units that the timer must execute. Each timing unit of the timers used in this experiment is $10 \mathrm{msec}$. In practical applications the stepper motor speeds can be in hundreds of steps per second. At such speeds it is not possible to see the stepping motion as the motor must stop for an instant before proceeding to the next step.

The Darlington amplifier interface was used in this experiment because the PLC voltages were too high. PLC system in general, provides high power from the Output Module that would normally be sufficient to drive directly larger motors.

PLCs are available with both the DC or AC power supplies. In the case of AC based PLCs, a considerable signal conditioning may be necessary to provide the proper drive signal for the stepper motor.

\section{Bibliography}

Introductory To Programmable Controllers, Mitsubishi Electric Corporation

Melsec Medoc Programming and Documentation Software, Mitsubishi Electronics America, Inc

FX Series Programmable Controllers Manual, Mitsubishi Electric Corporation

Analog and Digital Control Systems, R. Gayakwad/L. Sokoloff, Prentice Hall 1988

Programmable Logic Controllers, J. Webb/R. Reis, Prentice Hall 1995

Industrial Control Electronics, J. Webb/K. Greshock, Prentice Hall 1993

\section{Biography}

Leonard Sokoloff was born in Russia and immigrated to the United States in 1950 and was awarded the BSEE degree from Stevens Institute of Technology (1959), the MS Applied Science degree from Adelphi University (1964) and the PhDEE (candidate) from Stevens Institute of Technology. Worked in industry as semiconductor application and circuit design engineer (1959 - 1970). For the past 28 years with DeVRY Institute, currently as senior professor, teaching associate level and bachelor level courses in advanced mathematics and electrical engineering. 


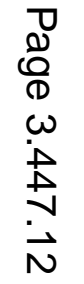

